\title{
DIVERSIDAD ESPACIO-TEMPORAL DE MACROINVERTEBRADOS COMO BIOINDICADORES DE LA CALIDAD DEL AGUA DEL RÍO YUQUIPA.
}

\section{Space-temporary diversity of macroinvertebrates as bioindicators of the water quality at Yuquipa river.}

\author{
${ }^{1}$ Patricio Méndez* (iD), ${ }^{1}$ Blanca Alvarez (iD), ${ }^{1}$ Nahomy Jaramillo (iD), ${ }^{2}$ Johanna Japa \\ ${ }^{1}$ Escuela Superior Politécnica de Chimborazo, Facultad de Ciencias/Ingeniería Ambiental, \\ Riobamba/Macas, Ecuador. \\ ${ }^{2}$ Investigador Independiente. \\ *patricio.mendez@espoch.edu.ec
}

$\mathrm{R}$ esumen

El estudio y desarrollo de las leyes ambientales que sistematizan el manejo de los cuerpos hídricos requiere la identificación de bioindicadores ara establecer su estado ecológico, es así que el presente estudio tiene como objetivo determinar la diversidad de macroinvertebrados como bioindicadores de la calidad del agua del río Yuquipa. Se estudiaron tres estaciones de monitoreo en los meses de noviembre y diciembre de 2020 y enero de 2021, en cada estación se recolectaron las especies de macroinvertebrados, el índice de Shannon se encargó de medir la diversidad de especies el cual expresa la uniformidad de los valores de importancia a través de todas las especies, en la determinación de la calidad del agua se utilizó el índice biótico de familia que asigna diferentes valores de tolerancia y considera el nivel taxonómico. Se reconocieron dos grupos de sitios. El primer grupo PY-2 y PY-3 fue valorado como calidad del agua excelente (3.26 y 3.12) respectivamente, con una diversidad media (2.45 y 2.54), mientras que en el sitio PY-1 fue valorado como calidad del agua regular (5.34) con una diversidad media de 2.36. Concluyendo que la diversidad y la calidad del agua no se han visto afectadas por las actividades antropogénicas.

Palabras claves: Índice biótico, hábitat fluvial, contaminación, equidad, abundancia, calidad del agua.

\section{A bstract}

The study and development of environmental laws that systematize the management of water bodies requires the identification of bioindicators to establish their ecological status. Therefore, this study aims to determine the macroinvertebrates diversity as bioindicators of Yuquipa river water quality. Three monitoring stations were studied on November, December 2020 and on January 2021; macroinvertebrates species were gathered and the Shannon index was used to measure species diversity, which expresses the uniformity of importance values across all species. Furthermore, the family biotic index was used to determine the water quality, as it ascribes different tolerance values and considers the taxonomic level. Two location groups were recognised: the first PY-2 and PY-3 was rated as excellent water quality (3.26 and 3.12) respectively, with a mean diversity (2.45 and 2.54), while the PY-1 site was rated as regular water quality (5.34) with a mean diversity of 2.36. The result was that diversity and water quality were not affected by anthropogenic activities.

Keywords: Biotic index, fluvial habitat, pollution, equity, abundance, water quality. 


\section{INTRODUCCIÓN}

En la actualidad existe gran interés en la determinación de la calidad del agua de las fuentes hídricas, siendo un factor determinante en el bienestar humano, ya que trae repercusiones en la salud de la población, el desarrollo económico y sobre todo la calidad ambiental de sus ecosistemas (1). Teniendo en cuenta que solo un $3,5 \%$ de la totalidad del agua existente en el planeta es agua dulce (2).

El control de la calidad del agua de los ecosistemas acuáticos se lleva a cabo mediante análisis físicos-químicos e indicadores biológicos como es el uso de macroinvertebrados que comprenden una gran parte de la diversidad acuática (3). La diversidad de especies es un parámetro muy importante en el estudio y descripción de comunidades, centrándose en la búsqueda de parámetros para su caracterización (4).

Los índices de diversidad de especies son formas matemáticas y sencillas de medir un conjunto de especies, combinando dos elementos de la estructura de las comunidades como es la riqueza y la equitabilidad (5). El índice que utilizó en esta investigación es el índice de Shannon- Wiener, el cual combina la información de la riqueza de especies y la equidad (6), Además cuantifica la diversidad específica, tomando en cuenta la cantidad de especies que existe en una muestra y la cantidad de individuos que hay para cada especie por tanto contempla la riqueza y la abundancia de las especies (7).

Para la investigación se ubicó tres puntos de muestreo en la zona alta, media y baja del río Yuquipa, el cual se origina en una laguna de selva virgen (8); ubicado en la parroquia "Sevilla Don Bosco" perteneciente al cantón Morona (9). Está zona se encuentra susceptible a cambios como la expansión agrícola, uso del suelo por asentamientos humanos los mismos generan contaminación por vertido de aguas residuales sin tratar efectuando cambios en la cantidad y calidad del recurso hídrico (10).

Es por ello que, hemos visto necesario determinar la calidad del agua mediante bioindicado- res como son los macroinvertebrados los cuales proporcionan excelentes señales sobre la calidad ambiental del agua de los ríos, porque algunos requieren de una muy buena calidad para desarrollarse y sobrevivir, mientras que otros, por el contrario, crecen y abundan en aguas muy contaminadas (11).

Este estudio se lo realizó con el objetivo de determinar la abundancia y diversidad de especies existentes en dicho rio utilizando el índice biótico de familias (IBF), el cual fue desarrollado y ejecutado en los EEUU por Hilsenhoff (1988). Este índice considera la diversidad de taxas, abundancia de las mismas y su función como bioindicadores; se ha elegido este método con el fin de determinar la calidad del agua de este afluente siendo este río el más representativo de la parroquia Sevilla Don Bosco puesto que es utilizado para las actividades de agricultura y ganaderías de la zona.

\section{MATERIALES Y MÉTODOS}

\section{Área de estudio}

Este estudio fue realizado en la parroquia Sevilla Don Bosco perteneciente a el cantón Morona, según el último censo realizado por el INEC (Instituto Nacional De Estadística Y Censo) la parroquia Sevilla Don Bosco cuenta con una población total de 16008 habitantes, esta parroquia tiene un área superficial de 2.306,54 Km2 y se encuentra ubicada al margen izquierdo del río Upano, planicie denominada Valle del Río Upano, frente a la ciudad de Macas, en las coordenadas $02^{\circ} 26^{\prime}$ de latitud sur y $78^{\circ} 11^{\prime}$ de longitud Oeste, se extiende desde los $400 \mathrm{msnm}$ hasta los $2300 \mathrm{msnm}$, con una temperatura promedio de $22{ }^{\circ} \mathrm{C}$.

El estudio se llevó a cabo en el rio Yuquipa en tres estaciones de muestreo diferentes cuyas coordenadas geográficas WGS84 se describe en la tabla 1 , se seleccionaron las tres estaciones debido a sus características, ya que en el punto 1 se encuentra localizado en el límite superior de la cabecera parroquial Sevilla Don Bosco, el punto 2 en medio de la cabecera parroquial Sevilla Don Bosco y el punto 3 en el límite inferior de la cabecera parroquial Sevilla Don Bosco. Este es 
uno de los ríos más representativos dentro de la parroquia puesto que los habitantes de esta zona hacen uso de este recurso hídrico y pueden llevar a cabo diversas actividades como, agricultura, recreación, consumo entre otras.

\begin{tabular}{|c|c|c|}
\hline \multicolumn{3}{|c|}{ COORDENADAS WGS 84 } \\
\hline ESTACIONES & $\mathbf{X}$ & $\mathbf{Y}$ \\
\hline PY-1 & 819912,75 & 9736098,75 \\
\hline PY-2 & 819711,94 & 9735548,00 \\
\hline PY-3 & 819659,18 & 9734791,08 \\
\hline
\end{tabular}

Tabla 1. Localización de las estaciones de muestreo

La toma de muestras se llevó a cabo en los meses de noviembre del 2020 a enero del año 2021, en cuanto a la obtención de los macroinvertebrados se establecieron tres puntos distintos de monitoreo los mismos que están distribuidos a lo largo del área de estudio y que comprende un tramo de $5 \mathrm{~km}$, al momento de escoger los puntos de muestreo se consideró la topografía del lugar, es decir, los puntos debían ser de fácil acceso, además se consideró la posición con respecto a la intervención de la zona urbana a lo largo del área de estudio, esto ayudó a determinar el estado y la calidad del agua.

\section{Recolección de muestras.}

Para la recolección de los macroinvertebrados se consideró una superficie de $15 \mathrm{~m} 2$ en cada uno de los puntos monitoreados, para ello se utilizó una red Surber la cual se sumergió a 90 grados a contracorriente y removiendo con las botas el lecho del río, el material removido se acumula en la red y con él los macroinvertebrados que haya en el sustrato, este procedimiento se lo realizó por un periodo de 60 minutos en cada punto de monitoreo. Una vez recaudados los macroinvertebrados con la ayuda de una bandeja de recolección y pinzas metálicas se procedió depositar en un recipiente de cristal el cual contenía alcohol al $80 \%$ para la preservación de la muestra y la posterior determinación taxonómica a nivel de familia de los individuos de acuerdo a las guías taxonómicas de Roldán (12) y Miñano et al., 2019 (13) mediante la utilización de un estereoscopio con características: Sistema Greenough de zoom óptico, cabezal binocular inclinado en $45^{\circ}$ con rango de giro de $360^{\circ}$, relación de zoom 04:01, rango ampliación: $1 \mathrm{X}-4 \mathrm{X}$ y sistema de enfoque grueso con ajuste de tensión. Esta actividad se realizó en el laboratorio de la "Escuela Superior Politécnica de Chimborazo Sede Morona Santiago”.

\section{Descripción de los puntos de muestreo}

PY-1 (Puente/ ingreso a la comunidad "Sagrado Corazón”): Inicio del tramo del rio en estudio, este punto fue considerado debido al hecho de que su caudal aún no ha sido intervenido por la zona urbana de la parroquia Sevilla Don Bosco.

PY-2 (Comunidades "Sagrado Corazón y San Luis de Inimkis"): Punto intermedio en el cual se pudo constatar que existe mayor intervención humana.

PY-3 (Río abajo): Punto final del tramo de estudio, este punto se localiza al margen de la parroquia y además se constata que no existen muchas viviendas que estén cerca de este sitio.

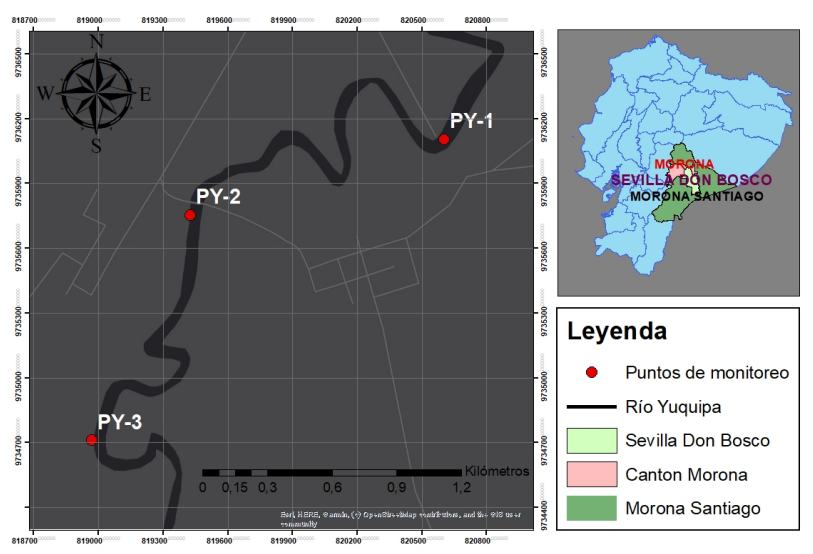

Figura 1. Área de estudio del Río Yuquipa.

Uno de los índices más utilizado y que se utilizó en este estudio para la determinación de la abundancia en los macroinvertebrados es el índice de Shannon- Wiener, el cual combina la información de la riqueza de especies y la equidad (14). Este se encarga de medir la diversidad de especies de plantas o animales en un determinado hábitat. Es utilizado para cuantificar la diversidad específica, tomando en cuenta la cantidad de especies que existe en una muestra y la cantidad de individuos que hay para cada especie por tanto contempla la riqueza y la abundancia de las especies (15).

Este índice sirve para expresar la uniformidad de los valores de importancia a través de todas las especies de muestras, midiendo el grado prome- 
dio, es decir, predice a que especie pertenece un individuo escogido al azar proveniente de una comunidad extensa (16).

Para el cálculo de la diversidad mediante el índice de Shannon se lo realiza aplicando la siguiente fórmula:

$$
H^{\prime}=-\sum \text { pi } \ln (p i)
$$

Donde:

pi $=$ Representa la proporción de individuos del total de la muestra que corresponde a la especie i. Se obtiene dividiendo ni/N.

ni $=$ número de individuos en el sistema correspondientes a la especie determinada $i$

$\mathrm{N}$ = número total de individuos de todas las especies en el sistema

$\ln =$ logaritmo natural

$\mathrm{S}=$ número total de especies

El rango de valores adquiridos en el índice de Shannon va entre 0 a 5 , cero cuando existe la presencia de una sola especie y el logaritmo natural $S$, cuando todas las especies se encuentran representadas por el mismo número de individuos (17). La valoración máxima es de 5 no obstante existen ecosistemas que pueden superar dicho valor, valores menores a 1,5 se considera como diversidad baja, valores entre 1,6 y 3 como diversidad media y por último valores iguales o mayores a 3,1 diversidad alta. Según los resultados del índice de Shannon ayudó a evaluar el área de estudio, si esta se encuentra en un mal estado de conservación ya sea por asentamiento humano, deforestación, actividad agrícola, indicándonos la presencia de especies de baja sensibilidad lo que quiere decir que el área se encuentra fragmentada y que las especies presentes son especies que se han ido adaptando a las condiciones actuales, ayudándonos así a determinar el grado de afectación del área (18).

\section{Índice de Hill}

La equitatividad es un reflejo del grado de constancia ambiental a que está sometida una comunidad fuertemente conectada con el ambiente abiótico, permite calcular el número efectivo de especies en una muestra, es decir, mide el número de especies cuando cada especie es ponderada por su abundancia relativa, se le calcula con la siguiente expresión.
$\mathrm{E}^{\prime}=\mathrm{N} 1 / \mathrm{N} 2$

Dónde:

$\mathrm{N} 1=$ número de especies abundantes $=\mathrm{eH}$

$\mathrm{N} 2=$ número de especies muy abundantes $=1 / \lambda$

Estos son los números de la serie de Hill, sin embargo, existen ocasiones en que este índice alcanza valores altos cuando la equidad es alta es decir que dos o más especies co-dominan la comunidad (19).

\section{Determinación de las familias abundantes}

Para la determinación de las familias más abundantes se considera la sumatoria final de las especies abundantes y se las divide por la riqueza especifica que presenta ese punto de monitoreo.

Familias abundantes $=\frac{\text { Eespecies abundantes }}{\text { Riqueza especifica }}$

A partir de esta ecuación obtendremos un número entero n, el cual va ser comparado con los valores de abundancia de cada familia, es decir $n$ $\geq$ abundancia.

\section{Índice biótico de familias}

El índice biótico de familia asignó diferentes valores de tolerancia, así mismo considera el nivel taxonómico de familia o genero de los organismos acuáticos (20).

Se contabilizaron los organismos de las diferentes familias recolectadas en cada sitio de muestreo, ponderando la abundancia de cada una de ellas al multiplicarlas por puntajes que indican el grado de sensibilidad a la contaminación (desde cero a diez, según se asocien a condiciones desde menor hasta mayor grado de contaminación orgánica), de esta manera se obtiene al final un promedio de la sumatoria (21).

Según (22) el IBF es un buen indicador para estimar la calidad de los ríos de cuencas agrícolas y ganaderas, además en la estimación de este índice se incorpora la relación entre la tolerancia y el total de familias encontradas en una muestra, lo cual, hace que el error de los muestreos cualitativos disminuya, permitiendo que sea factible su cálculo (23).

Se obtiene mediante la siguiente ecuación. 


$$
I B F=\frac{\sum_{i=1}^{n}(X i * T i)}{\sum_{i=1}^{n} n i}
$$

Donde:

$\mathrm{Xi}=$ Es el número de individuos en una familia/ género.

$\mathrm{Ti}=$ Es el puntaje de tolerancia de cada familia/ género.

ni $=$ Es el número total de individuos en cada estación.

Para la determinación de este índice se estableció una tabla donde detalla los valores de tolerancia.

\begin{tabular}{|c|c|c|c|c|c|}
\hline ORDEN O CLASE & FAMILA & VALOR DE TOLERANCIA & ORDEN O CLASE & FAMILIA & VALOR DE TOLERANCIA \\
\hline \multicolumn{2}{|l|}{ Plecoptera } & & \multicolumn{2}{|l|}{ Megaloptera } & \\
\hline & Gripopterygiidae & 1 & & Corydalidae & 0 \\
\hline & Nolonemouridae & 0 & & Sialidae & 4 \\
\hline & Perlidae & 1 & \multicolumn{2}{|l|}{ Lepidoptera } & \\
\hline & Diamphipnoidae & 0 & & Pyralidae & 5 \\
\hline & Eustheniidae & 0 & Platyhelminthes & & \\
\hline & Austroperlidae & 1 & & Turbellaria & 4 \\
\hline \multicolumn{2}{|l|}{ Ephemeroptera } & & \multicolumn{2}{|l|}{ Acari } & 4 \\
\hline & Baetidae & 4 & \multicolumn{2}{|l|}{ Decapoda } & 6 \\
\hline & Caenidae & 7 & \multicolumn{2}{|l|}{ Coloptera } & \\
\hline & Leptophlebiidae & 2 & & \multirow{2}{*}{ Elmidae } & \multirow{2}{*}{4} \\
\hline & Leptohyphidae & 6 & & & \\
\hline & Siphlonuridae & 7 & & Psephenidae & 4 \\
\hline & Oligoneuridae & 2 & \multicolumn{2}{|l|}{ Diptera } & \\
\hline & Amelotopsidae & 2 & & Athericidae & 2 \\
\hline & Coloburiscidae & 3 & & Blephariceridae & 0 \\
\hline & Oniscigastridae & 3 & & Ceratopogonidae & 6 \\
\hline \multicolumn{2}{|l|}{ Odonata } & & & Chirinomidae & 7 \\
\hline & Aeshinidae & 3 & & Empididae & 6 \\
\hline & Calopterygidae & 5 & & Ephydridae & 6 \\
\hline & Gomphidae & 1 & & Psychodidae & 10 \\
\hline & Lestidae & 9 & & Simuliidae & 6 \\
\hline & Libellulidae & 9 & & Tipulidae & 3 \\
\hline & Coenagrionidae & 9 & \multicolumn{2}{|l|}{ Amphipoda } & \\
\hline & Cordulidae & 5 & & Gammaridae & 4 \\
\hline & Petaluridae & 5 & & Hyalellidae & 8 \\
\hline & Calomoceratidae & 3 & \multicolumn{2}{|l|}{ Mollusca } & \\
\hline & Glossosomatidae & 0 & & Amnicolidae & 6 \\
\hline & Helicopsychidae & 3 & & Lymnaeidae & 6 \\
\hline & Hydropsychidae & 4 & & Physidae & 8 \\
\hline & Hydroptilidae & 4 & & Sphaeriidae & 8 \\
\hline & Leptoceridae & 4 & & Chilinidae & 6 \\
\hline & Limnephilidae & 2 & \multicolumn{2}{|l|}{ Oligochaeta } & 8 \\
\hline & Ecnomidae & 3 & \multicolumn{2}{|l|}{ Hirudenea } & 10 \\
\hline & Helicophidae & 6 & Decapoda & Palaemonidae & 4 \\
\hline & Polycentropodidae & 3 & & & \\
\hline & Philopotamidae & 2 & & & \\
\hline & Hydrobiosidae & 0 & & & \\
\hline & Sericostomatidae & 3 & & & \\
\hline
\end{tabular}

Tabla 2. Valores de tolerancia de macroinvertebrados bentónicos utilizados en la determinación de Índice Biótico de Familias (IBF). Fuente (24) 
Los valores del índice biótico de familia se detallan mediante siete clases de calidad ambiental, correspondiendo a una escala biológica, la cual fue desarrollada para determinar y evaluar el grado de contaminación o perturbación del ambiente evaluado.

\begin{tabular}{|c|c|l|l|}
\hline Valor IBF & Categoría & $\begin{array}{l}\text { Calidad del } \\
\text { Agua }\end{array}$ & $\begin{array}{l}\text { Interpretación del grado de } \\
\text { contaminación Orgánica }\end{array}$ \\
\hline $\mathbf{0 , 0 0}-\mathbf{3 , 7 5}$ & $\mathbf{1}$ & Excelente & $\begin{array}{l}\text { Contaminación orgánica } \\
\text { improbable }\end{array}$ \\
\hline $\mathbf{3 , 7 6}-\mathbf{4 , 2 5}$ & $\mathbf{2}$ & Muy Buena & $\begin{array}{l}\text { Contaminación orgánica } \\
\text { leve posible }\end{array}$ \\
\hline $\mathbf{4 , 2 6 - 5 , 0 0}$ & $\mathbf{3}$ & Buena & $\begin{array}{l}\text { Alguna contaminación } \\
\text { orgánica probable }\end{array}$ \\
\hline $\mathbf{5 , 0 1}-\mathbf{5 , 7 5}$ & $\mathbf{4}$ & Regular & $\begin{array}{l}\text { Contaminación orgánica } \\
\text { bastante sustancial es pro- } \\
\text { bable }\end{array}$ \\
\hline $\mathbf{5 , 7 6 - 6 , 5 0}$ & $\mathbf{5}$ & $\begin{array}{l}\text { Regular } \\
\text { Pobre }\end{array}$ & $\begin{array}{l}\text { Contaminación sustancial } \\
\text { probable }\end{array}$ \\
\hline $\mathbf{6 , 5 1}-\mathbf{7 , 2 5}$ & $\mathbf{6}$ & Pobre & $\begin{array}{l}\text { Contaminación sustancial } \\
\text { muy probable }\end{array}$ \\
\hline $\mathbf{7 , 2 6 -}$ & 7 & Muy pobre & $\begin{array}{l}\text { Contaminación orgánica } \\
\text { severa probable }\end{array}$ \\
\hline $\mathbf{1 0 , 0 0}$ & 7 &
\end{tabular}

Tabla 3. Clasificación de la Calidad de Agua según el Índice Biótico de Familias (IBF). Fuente: (23)

\section{RESULTADOS}

Durante el estudio se recolectaron un total de 908 macroinvertebrados acuáticos, pertenecientes a 9 órdenes, entre las cuales destacan 5 órdenes con 7 familias abundantes en los tres puntos de monitoreo, como se detalla en la tabla 4.

\begin{tabular}{|c|c|c|c|}
\hline \multicolumn{3}{|c|}{ ESTACIÓN PY-1 } \\
\hline$\#$ & Orden & Familia & $\begin{array}{c}\text { Especies Abundantes } \\
\text { (Abundancia total) }\end{array}$ \\
\hline 1 & Díptera & Chironomidae & 33 \\
\hline 2 & Ephemeroptera & Leptohyphidae & 35 \\
\hline 3 & Ephemeroptera & Leptophlebiidae & 53 \\
\hline 4 & Odonata & Libellulidae & 27 \\
\hline
\end{tabular}

Tabla 4. Resultados de familias abundantes en el punto PY-1.

\begin{tabular}{|c|c|c|c|}
\hline \multicolumn{3}{|c|}{ ESTACIÓN PY-2 } \\
\hline$\#$ & Orden & Familia & $\begin{array}{c}\text { Especies Abundantes } \\
\text { (Abundancia total) }\end{array}$ \\
\hline 1 & Odonata & Calopterygidae & 26 \\
\hline 2 & Trichóptero & Hydropsychidae & 23 \\
\hline 3 & Ephemeroptera & Leptohyphidae & 46 \\
\hline 4 & Ephemeroptera & Leptophlebiidae & 62 \\
\hline 5 & Decápoda & Palaemonidae & 26 \\
\hline
\end{tabular}

Tabla 5. Resultado de familias abundantes en el punto PY-2.

\begin{tabular}{|c|c|c|c|}
\hline \multicolumn{3}{|c|}{ ESTACIÓN PY-3 } \\
\hline$\#$ & Orden & Familia & $\begin{array}{c}\text { Especies Abundantes } \\
\text { (Abundancia total) }\end{array}$ \\
\hline 1 & Odonata & Calopterygidae & 37 \\
\hline 2 & Trichóptero & Hydropsychidae & 20 \\
\hline 3 & Ephemeroptera & Leptohyphidae & 53 \\
\hline 4 & Ephemeroptera & Leptophlebiidae & 77 \\
\hline 5 & Decápoda & Palaemonidae & 36 \\
\hline
\end{tabular}

Tabla 6. Resultado de familias más abundantes en el punto PY-3

El orden Ephemeroptera de la familia Leptophlebiidae es uno de los macroinvertebrados acuáticos más abundantes con 192 especies, en el mismo orden, pero en la familia Leptohyphidae se encontró 134 especies, en el orden Odonata se encontró dos familias la Calopterygidae con 63 especies y la familia Libellulidae con 27 especies, en el orden decápoda en la familia palaemonidae se encontró 62 especies seguido de la orden Trichóptero de la familia Hydropsychidae con 43 especies y por último el orden Díptera con la familia Chironomidae la cual tiene 33 especies; todas estas especies se recolectaron a lo largo de los tres puntos encontrandoce diferencias entre sus abundancias debido a su diferente cobertura vegetal a la ribera del río.

\begin{tabular}{|c|c|c|c|}
\hline INDICES & $\begin{array}{c}\text { ESTACIÓN } \\
\text { PY-1 }\end{array}$ & $\begin{array}{c}\text { ESTACIÓN } \\
\text { PY-2 }\end{array}$ & $\begin{array}{c}\text { ESTACIÓN } \\
\text { PY-3 }\end{array}$ \\
\hline Riqueza & 14 & 15 & 18 \\
\hline Especies abundantes & 246 & 298 & 364 \\
\hline Índice de Shannon & 2,36 & 2,46 & 2,54 \\
\hline Equidad de Hill & 1,23 & 0,81 & 0,76 \\
\hline
\end{tabular}

Tabla 7. Riqueza, Índice de Shannon, equidad del Hill y especies abundantes en cada estación de muestreo.

A nivel de riqueza se puede observar según la tabla 7, que en la estación de muestreo PY-3 fue mayor con un valor de 18 a diferencia de la estación PY-1 y PY-2 que tienen valores de 14 y 15 respectivamente, sin embargo, para la determinación de especies abundantes se pudo constatar que existió una mayor presencia de macroinvertebrados acuáticos en la estación PY-3.

Para el cálculo del índice de Shannon se consideró todas las especies recolectadas en cada punto de muestreó las cuales se encuentran detalladas en la tabla 4, 5 y 6 teniendo como resultado una diversidad media en cada uno de los puntos puesto que según (Alvarez, E 2016) el rango 
establecido va de 0 a 5 siendo 5 una valoración máxima en este caso los resultados obtenidos se encontraron en un rango de 1,6 a 3 indicando una diversidad media en los tres puntos; al calcular la equidad de Hill se pudo constatar en los puntos PY-2 y PY-3 existe una mayor diversidad debido a que los valores de la equidad según Hill son cercanos a cero, sin embargo, en el PY-1 existe menor diversidad por que la equidad es mayor a uno.

\begin{tabular}{|c|c|c|c|c|c|}
\hline ORDEN & FAMILIA & ABUNDANCIA & PUNTAJE & ABD $^{\star}$ PTJ & $\left(\right.$ ABD $^{\star}$ PTJ) /TOTAL \\
\hline Díptera & Chironomidae & 33 & 7 & 231 & 1,56081081 \\
\hline Ephemeroptera & Leptohyphidae & 35 & 6 & 210 & 1,41891892 \\
\hline Ephemeroptera & Leptophlebiidae & 53 & 2 & 106 & 0,71621622 \\
\hline Odonata & Libellulidae & 27 & 9 & 243 & 1,64189189 \\
\hline ABUN. TOTAL & & 148 & IBF & & 5,34 \\
\hline
\end{tabular}

Tabla 8. Cálculos del Índice Biótico de Familias (IBF)

\begin{tabular}{|c|c|c|c|c|c|}
\hline ORDEN & FAMILIA & ABUNDANCIA & PUNTAJE & $A B D^{\star P T J}$ & $\left(\mathrm{ABD}^{\star}{ }^{\mathrm{PTJ}}\right) / \mathrm{TOTAL}$ \\
\hline Odonata & Calopterygidae & 26 & 5 & 130 & 0,71038251 \\
\hline Trichóptero & Hydropsychidae & 23 & 4 & 92 & 0,50273224 \\
\hline Ephemeroptera & Leptohyphidae & 46 & 6 & 276 & 1,50819672 \\
\hline Ephemeroptera & Leptophlebiidae & 62 & 2 & 124 & 0,67759563 \\
\hline Decápoda & Palaemonidae & 26 & 4 & 104 & 0,56830601 \\
\hline ABUN. TOTAL & & 183 & IBF & & 3,26 \\
\hline
\end{tabular}

Tabla 9. Cálculos del Índice Biótico de Familias (IBF)

\begin{tabular}{|c|c|c|r|r|r|}
\hline ORDEN & FAMILIA & ABUNDANCIA & PUNTAJE & ABD ${ }^{\star}$ PTJ & (ABD ${ }^{\star}$ PTJ) /TOTAL \\
\hline Odonata & Calopterygidae & 37 & 5 & 185 & 0,82959641 \\
\hline Trichóptero & Hydropsychidae & 20 & 4 & 80 & 0,35874439 \\
\hline Ephemeroptera & Leptohyphidae & 53 & 6 & 318 & 1,42600897 \\
\hline Ephemeroptera & Leptophlebiidae & 77 & 2 & 154 & 0,69058296 \\
\hline Decápoda & Palaemonidae & 36 & 4 & 144 & 0,64573991 \\
\hline ABUN. TOTAL & & & 223 & IBF & \\
\hline
\end{tabular}

Tabla 10. Cálculos del Índice Biótico de Familias (IBF)

Para el cálculo del IBF se considero las especies más abundantes por cada punto tomando en cuenta los valores de tolerancia de macroinvertebrados bentónicos propuesta por Hilsenhoff, los resultados se encuentra detallados en las tablas 8,9 y 10.

Se puede observar en la tabla 8 los resultados de la estación de muetreo PY-1 tiene una calidad del agua regular con una puntuación de 5,34 la cual corresponde a una escala biologica que va desde 5,01 a 5,75 y determina el grado de contaminacion o perturbacion del punto de muestreo a evaluar, según la clasificación de la calidad del agua del IBF en el punto de muestreo es probable que exista una contaminación orgánica bastante sustancial.

Para las estaciones de monitoreo PY-2 y PY-3 los resultados fueron de 3,26 y 3,12 respectivamen- te, lo cual nos indica que la calidad del agua en esos dos puntos es excelente y de acuerdo a la clasificación de la calidad del agua según el IBF existe una contaminación orgánica improbable.

\section{DISCUSIÓN}

En cuanto a la riqueza específica, se acoge la propuesta de Moreno (19), quien al igual que nuestro estudio determinó que un indicador del estado del hábitat, basado en la hipótesis de que un tramo heterogéneo, con alta calidad del agua, permitirá la existencia de una mayor diversidad de especies, siendo así que el punto PY-3 el cual tiene una calidad del agua excelente presenta una mayor diversidad que PY-2 y PY-1 siendo este último un tramo afectado por acciones antropogénicas.

Los trechos estudiados en el río Yuquipa descri- 
ben una homogeneidad espacial, en los cuales se denota que en la parte baja de la zona de estudio (PY-2 y PY-3) un dominante potencial de hojarasca y de sustratos rocosos, lo cual influyeron en la mayor abundancia y riqueza lo que coincide con lo expresado por Walteros., et al (25), y Morelli (26)

Se han comparado varios estudios realizados en ríos de colombianos por Nuñéz (27), Walteros., et al (25), y Roldan (28), con nuestro estudio y se ha podido constatar que existe una riqueza especifica y una diversidad alta, además existe una predominancia de los órdenes Ephemeroptera y Odonata encontrados a lo largo de los puntos de muestreo, esto se relaciona con la existencia de aguas transparentes con elevada oxigenación lo que favorece las características físicas del lugar de estudio.

Según Damanik et al., (29) la determinación de la calidad del agua se encuentra en relación a la proporción y abundancia de macroinvertebrados, esto se ve evidenciado en los resultados de nuestro estudio, pues la estaciones que mayor abundancia de especies presentaron PY-2 y PY-3 obtuvieron una calidad del agua excelente según la metodología utilizada.

\section{CONCLUSIONES}

Se registraron 908 macroinvertebrados acuáti- cos distribuidos en 9 órdenes y 19 familias a lo largo del tramo del rio Yuquipa, el punto de muestreo que mayor riqueza tuvo fue el PY-3 el mismo que tiene un valor de 18 , a diferencia de los puntos de muestreo PY-2 y PY-1 poseen una baja riqueza, esto se debe a la intervención humana y la contaminación que se produce dado que los macroinvertebrados son muy sensibles y no se adaptan al cambio ocurrido, mientras que para la determinación de especies abundantes se pudo constatar que existió una mayor presencia de macroinvertebrados acuáticos en la estación PY-3 con 364 especies.

Para el índice de Shannon el resultado fue una diversidad media en cada uno de los puntos de muestreo y para la equidad de Hill se pudo constatar en los puntos PY-2 y PY-3 existe una mayor equidad debido a que los valores son cercanos a cero enfocándose en el grado de dominancia y la distribución de las especies

Por ende, podemos concluir que la diversidad de macroinvertebrados bentónicos y la calidad de agua en las estaciones muestreadas del rio $\mathrm{Yu}$ quipa no se han visto afectadas por las actividades antropogénicas. Además podemos finalizar diciendo que el agua de esta fuente hídrica que abastece a gran parte de la población de la parroquia Sevilla Don Bosco presenta buena calidad, evidenciada por los valores del índice biótico de familias IBF.

$\mathrm{R}$ eferencias

1. Cazorla X. Propuesta De Diseño De Un Sistema De Tratamiento Biológico De Aguas Residuales De La Comunidad "El Tejar Balbanera " En Guamote". Interciencia. 2018;489(20):313-35.

2. Triyanto J, Janjua PZ, Samad G, Khan N, Ishaq M, Rumiati AT, et al. EValuación De La Calidad Del Agua Mediante La Utilización De Macro Invertebrados Bentónicos, Como Bioindicadores: Estudio De Caso En El Río Suárez (Chiquinquirá - Boyacá). Rev Chil Hist Nat. 2003;6(1):51-66.

3. Ladrera R. Los macroinvertebrados acuáticos como indicadores del estado ecológico de los ríos. Páginas Inf Ambient [Internet]. 2018;39:24-9. Disponible en: https://dialnet.unirioja.es/descarga/articulo/4015812. pdf

4. Hauer R, Lamberti G. Métodos en ecología de arroyos. Estructura del ecosistema. Nueva York: Elsevier Inc.; 2017. 128 p.

5. García S, Moreno C, Morón M, Castellanos I, Pavón N. Integrando la estructura taxonómica en el análisis de la diversidad alfa y beta de los escarabajos Melolonthidae en la Faja Volcánica Transmexicana. Rev Mex Biodivers [Internet]. 2016;87(3):1033-44. Disponible en: http://dx.doi.org/10.1016/j.rmb.2016.06.003

6. González M, Zaragoza S, Pérez C. Análisis de la diversidad de Coleoptera en el bosque tropical caducifolio en Acahuizotla, Guerrero, México. Rev Mex Biodivers [Internet]. 2017;88(2):381-8. Disponible en: http://dx.doi.org/10.1016/j.rmb.2017.03.008 
7. Jost L, Gonz A. Midiendo la diversidad Biológica: Más alla del índice de Shannon. Acta zoológica Lilloana. 2012;56(January 2012):14.

8. Rodríguez H. Plan Estrategico de desarrollo turistico sostenible para la parroquia Sevilla Don Bosco, cantón Morona, provincia de Morona Santiago 2015-2025. 2016;151.

9. Durán P. Memoria Tecnica Alcantarillado Subsistema 12. Alcaldia Morona. Macas; 2015.

10. Valladolid M, Martínez-Bastida JJ, Araúzo M, Gutiérrez C. Abundancia y biodiversidad de los macroinvertebrados del río Oja (La Rioja, España). Limnetica. 2006;25(3):745-52.

11. Bernal J, Rodriguez A, Rios T. Contribuciones Al Desarrollo De La Entomología En Panamá. Puente Biol. 2019;9:55-67.

12. Roldán G. Bioindicador de la calidad del agua en Colombia; Propuesta para el uso del método BMWP/ Col. Antioquia: Universidad de Antioquia; 2003.

13. Miñano P, Olaya M, Huamantinco AA. Clave taxonómica de ninfas de Ephemeroptera (Insecta) del sudeste de Perú. Rev Peru Biol. 2019;26(4):411-28.

14. Vinhote E, Freitas F, Azevedo C, Souza C. Diversity and similarity of species of natural regeneration after logging in commercially managed forest in central amazon. Cienc Florest. 2020;30(4):1116-29.

15. Damanik-Ambarita MN, Lock K, Boets P, Everaert G, Nguyen THT, Forio MAE, et al. Ecological water quality analysis of the Guayas river basin (Ecuador) based on macroinvertebrates indices. Limnologica [Internet]. marzo de 2016;57:27-59. Disponible en: https://linkinghub.elsevier.com/retrieve/pii/ S0075951116000050

16. Campo AM, Duval VS. Diversidad y valor de importancia para la conservación de la vegetación natural. Parque Nacional Lihué Calel (Argentina). An Geogr la Univ Complut. 2014;34(2):25-42.

17. Rubio S. Cálculo del índice de biodiversidad de especies faunísticas en el Bosque Protector Aguarongo. Tesis. 2016;73.

18. Alvarez E. Informe del estado actual del componente biótico al proyecto de Estudio de Impacto Ambiental y Plan de Manejo Ambiental del Puerto de Aguas Profundas de Posorja. Cardno. 2016;6-192.

19. Moreno C. Métodos para medir la biodiversidad. Manuales y Tesis SEA. Zaragoza-España; 2001. 86 p.

20. Huaman Matos LD. Diversidad de macroinvertebrados indicadores de calidad de agua en las lagunas de Pucushuclo y Nahuimpuquio - Provincia de Chupaca. 2019;109.

21. Sermeño J, Serrano L, Springer M, Paniagua M, Pérez D, Rivas A, et al. Determinación de la calidad ambiental de las aguas de los ríos de El Salvador, utilizando invertebrados acuáticos. Vol. 1, Bioma. San Salvador, El Salvador; 2010.

22. Figueroa R, Palma A, Ruíz V, Niell X. Análisis comparativo de índices bióticos utilizados en la evaluaciónde la calidad de las aguas en un río mediterráneo de Chile:río Chillán, VIII Región. Rev Chil Hist Nat. 2007;225-242(225-242):18.

23. Cárdenas E, Lugo L, González J, Tenjo A. Aplicación del indice biotico de familias de Macroinvertebrados Para La Caracterización Del Río Teusaca, afluente del rio Bogotá. UDCA Actual Divulg Científica. 2018;21(2):587-97.

24. Figueroa R, Valdovinos C, Araya E, Parra O. Macroinvertebrados bentónicos como indicadores de calidad de agua de ríos del sur de Chile. En: Revista Chilena de Historia Natural. Sociedad de Biología de Chile; 2003. p. 275-85.

25. Rodríguez JMW, Rojas JMC, Gómez JHM. Ensamble de macroinvertebrados acuáticos y estado ecológico de la microcuenca Dalí-Otún, Departamento de Risaralda, Colombia. Hidrobiologica. 2016;26(3):35971.

26. Morelli E, Verdi A. Diversidad de macroinvertebrados acuáticos en cursos de agua dulce con vegetación ribereña nativa de Uruguay. Rev Mex Biodivers. 2014;85(4):1160-70.

27. Nuñez JC, Fragoso P. Uso de macroinvertebrados acuáticos como bioindicadores de la calidad del agua en la cuenca media del rio Guatapurí (Valledupar, Colombia). Inf tecnológica [Internet]. 2020;31(6):207-16. Disponible en: http://www.scielo.cl/scielo.php?script=sci_arttext\&pid=S0718-07642020000600207\&lan$\mathrm{g}=\mathrm{es} \% 0 \mathrm{Ahttp}: / /$ www.scielo.cl/pdf/infotec/v31n6/0718-0764-infotec-31-06-207.pdf

28. Roldán-Pérez G. Los macroinvertebrados como bioindicadores de la calidad del agua: cuatro décadas de desarrollo en Colombia y Latinoamerica. Rev la Acad Colomb Ciencias Exactas, Físicas y Nat. 2016;40(155):254.

29. Damanik M, Pieter C, Tien G, Eurie M, Peace S, Suhareva N, et al. Ecological water quality analysis of the Guayas river basin (Ecuador)based on macroinvertebrates indices. Limnologica. 2016; 